\title{
喹唑啉-4-酮衍生物的合成及抗肿瘤活性
}

\author{
孙佳婧周利凯谭官海李帅王淑霞 \\ 陈华* 李小六*
}

(河北大学化学与环境科学学院 河北省化学生物学重点实验室 保定 071002)

\begin{abstract}
摘要 以邻氨基苯甲酸、芳醛和甘氨酸乙酯为原料, 利用亚胺和亚胺烯酮的加成反应, 芳构化合成了系列含氨基酸链 的 $C-2$ 和 $N-3$ 双取代的喹唑啉-4-酮衍生物 5. 酸性条件下脱除羧甲基, 设计合成了连有氨基侧链的喹唑啉酮衍生物 9, 并评价了化合物的抗肿瘤细胞增殖活性. 化合物 $9 \mathbf{b a}$ 和 $9 \mathrm{bc}$ 具有较好的抗 Hela 细胞增殖活性, $\mathrm{IC}_{50}$ 值分别为 8.16 和 7.47 $\mu \mathrm{mol} / \mathrm{L}$, 而 $9 \mathrm{bc}$ 和 $9 \mathrm{bd}$ 具有较好的抗 A549 细胞增殖活性, $\mathrm{IC}_{50}$ 值分别为 5.62 和 $9.54 \mu \mathrm{mol} / \mathrm{L}$. 构效关系表明, $C-2$ 位香豆 素(较大的 $\pi$ 平面芳环)取代以及氨基侧链的引入有利于化合物的抗肿瘤活性.

关键词＼cjkstart喹唑啉-4-酮; 抗肿瘤活性; 亚胺烯酮; 氨基侧链; 羧甲基脱除; 香豆素
\end{abstract}

\section{Synthesis and Anti-tumor Activity of Novel Quinazolin-4-one Derivatives}

\author{
Sun, Jiajing \\ Zhou, Likai \\ Tan, Guanhai \\ Li, Shuai \\ Wang, Shuxia
}

Chen, Hua* Li, Xiaoliu*

(Key Laboratory of Chemical Biology of Hebei Province, College of Chemistry and Environmental Science,

Hebei University, Baoding 071002)

\begin{abstract}
A series of novel $C-2$ and $N-3$ disubstituted quinazolin-4(3H)-one derivatives 5 possessing amino acid chain were synthesized by the addition reaction of imine with iminoketene and aromatization using anthranilic acid, aromatic aldehyde and glycine ethyl ester as starting materials. After elimination of carboxymethyl in the acidic condition, novel quinazolin-4(3H)-one derivatives 9 bearing amino side chain were designed and synthesized by SN2 substitution reaction. The compounds were evaluated for their anti-proliferation activities against four tumor cells. The results showed that some of the compounds, such as $\mathbf{9 b a}$ and $9 \mathrm{bc}$ showed significantly anti Hela activity with the $\mathrm{IC}_{50}$ values of $8.16 \mu \mathrm{M}$ and $7.47 \mu \mathrm{M}$, respectively, while $9 \mathbf{b c}$ and $9 \mathbf{b d}$ against $\mathrm{A} 549$ with the $\mathrm{IC}_{50}$ values of $5.62 \mu \mathrm{M}$ and $9.54 \mu \mathrm{M}$, respectively. Structure activity relationship analysis of these analogues suggested that coumarin substituent (extended $\pi$ planar aromatic ring) on $C-2$ position and the introduction of amino side chain should be favorable to their anti-tumor activities.
\end{abstract}

Keywords quinazolin-4-one; anti-tumor activity; iminoketene; amino side chain; elimination of carboxymethyl; coumarin

喹唑啉-4-酮是一类广谱的药效基团，其衍生物多 具有抗菌、抗炎、抗疮疾、抗结核、抗肿瘤和抗病毒等 作用 ${ }^{[1]}$. 其中 $C-2$ 和 $N-3$ 取代基的结构各异，赋予了喹唑 啉-4-酮衍生物多样的生物活性 ${ }^{[2]}$. 因此, 喹唑啉-4-酮的 合成及生物活性研究备受关注 ${ }^{[3,4]}$. 目前, 喹唑啉-4-酮 衍生物的合成主要有两种(Scheme 1): 其一是以邻氨基 苯甲酰胺与醛反应, 以 $C-2$ 位单取代产物为主, 收率较
高 ${ }^{[5,6]}$; 其二是以邻氨基苯甲酸为起始原料, 先制备其 苯并噁嗪-4-酮衍生物, 进而胺解得到 $C-2$ 和 $N-3$ 位双取 代产物 ${ }^{[7,8]}$. 此路线分两步进行，易于制备 $N-3$ 位取代基 改变的产物, 但在获得结构更加多样的产物时, 略显繁 琐.

近年来, 文献报道通过邻氨基苯甲酸与亚胺(Schiff base)的一锅法反应，可以简便合成 $C-2$ 和 $N-3$ 双取代喹

*Corresponding authors. E-mail: lix1@hbu.cn; hua-todd@163.com

Received July 29, 2016; revised September 13, 2016; published online October 9, 2016.

Project supported by the National Natural Science Foundation of China (No. 21372060), the Hebei Provincal Natural Science Fund for Distinguished Young Scholars (Incubation) (No. B2015201005).

国家自然科学基金(No. 21372060)、河北省自然科学杰出青年基金(培育)(No. B2015201005)资助项目. 
<smiles>NC(=O)c1ccccc1NC(=O)OCc1ccccc1</smiles>

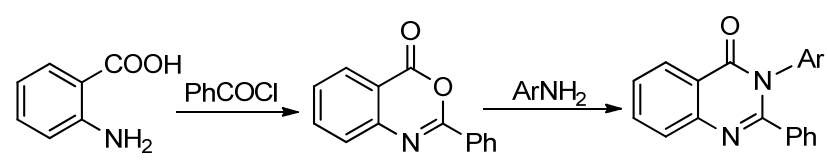

图式 1 喹唑啉-4-酮的合成方法

Scheme 1 Synthetic protocols for quinazolin-4(3H)-one

唑啉-4-酮衍生物 ${ }^{[9]}$. 由于邻氨基苯甲酸中氨基的亲核性 较弱, 使得氨基直接对亚胺的亲核加成难以发生. 因此, 该法首先将邻氨基苯甲酸转化成其相应的亚胺烯酮中 间体, 进而与亚胺加成缩合得到目标产物 ${ }^{[10,11]}$. 此法原 料易得且多样, 为喹唑啉-4-酮 $C-2 、 N-3$ 双取代产物的 制备提供了一个有效的便利方法. 本文利用此法, 以邻 氨基苯甲酸、苯甲醛(或香豆素醛)和甘氨酸乙酯为原料, 简便地合成了含氨基酸链的二氢喹唑啉-4-酮衍生物 4 (Scheme 2).
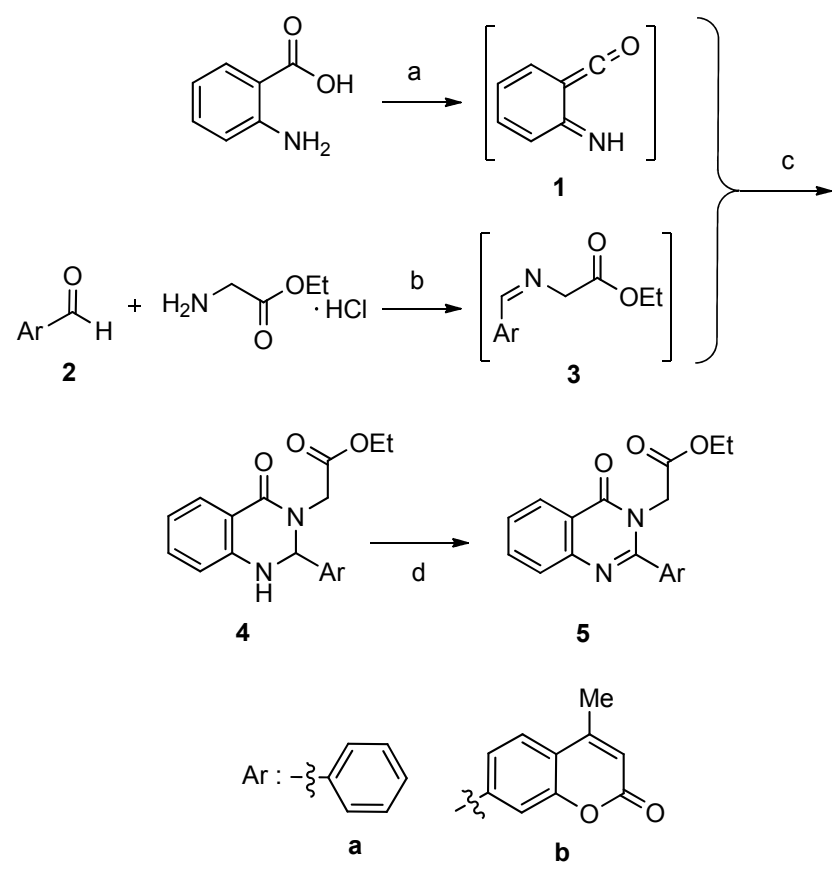

Reagents and conditions: (a) dry benzene, $\mathrm{SOCl}_{2}$, reflux, $3 \mathrm{~h}$; (b) dry benzene, DIPEA, r.t., 4 h; (c) dry benzene, r.t., 8 h; (d) acetone, $\mathrm{KMnO}_{4}$, reflux, $3 \mathrm{~h}$

图式 2 化合物 $\mathbf{4}$ 和 $\mathbf{5}$ 的合成

Scheme 2 Synthesis of compounds 4 and 5

\section{1 结果与讨论}

\section{1 喹唑啉-4-酮环的构建}

如 Scheme 2 所示, 邻氨基苯甲酸在氯化亚砜作用 下得到亚胺烯酮中间体 1 . 以苯甲醛 $(2 a)$ 和甘氨酸乙酯 盐酸盐为原料, 碱性条件下合成相应的亚胺中间体 $\mathbf{3 a}$,
催化量的冰醋酸有利于亚胺的生成. 不经分离, 亚胺中 间体 3a 直接与亚胺烯酮中间体 1 反应，得到二氢喹唑 啉-4-酮衍生物 4a. 我们对亚胺与亚胺烯酮的加成反应 条件进行了简单探讨, 改变投料比例、升高温度、延长 反应时间对产率影响并不明显(表 1). 最终确定最佳反 应条件是以苯为溶剂, 室温反应 $8 \mathrm{~h}$, 以 $72 \%$ 的收率得 到了含氨基酸链的二氢喹唑啉-4-酮衍生物 4a. 需要指 出的是, 反应过程中会有少量氧化产物 $\mathbf{5 a}$ 的生成, 而且 混合体系下 $4 \mathrm{a}$ 不分离直接芳构也可以得到 $5 \mathrm{a}$. 但考虑 到后续实验中 $5 \mathrm{a}$ 难以分离, 故在此步快速柱分离并重 结晶得到 4a. 在高锰酸钾的作用下, $4 \mathbf{a}$ 以几乎定量的收 率得到 5a, 反应操作更为简便. 利用同样的方法, 以 7香豆素醛 $\mathbf{2 b}^{[12]}$ 为原料, 得到了化合物 $\mathbf{5 b}$. 此方法将可 用于更多 $C-2$ 和 $N-3$ 双取代喹唑啉-4-酮衍生物的合成.

表 1 二氢喹唑啉-4-酮(4a)的合成

Table 1 Synthesis of dihydroquinazolin-4-one (4a)

\begin{tabular}{ccccc}
\hline Entry & 投料比 $^{a}$ & 温度 $/{ }^{\circ} \mathrm{C}$ & 时间 $/ \mathrm{h}$ & 收率 $/ \%$ \\
\hline 1 & $1: 1$ & r.t & 8 & 45 \\
2 & $1: 1$ & 40 & 8 & 47 \\
3 & $1: 1$ & 80 & 8 & 40 \\
4 & $1: 1$ & r.t & 10 & 48 \\
5 & $1: 1$ & r.t & 12 & 47 \\
6 & $1: 2$ & r.t & 8 & 48 \\
7 & $1: 5$ & r.t & 8 & 52 \\
$8^{b}$ & $1: 1$ & r.t & 8 & 72 \\
\hline
\end{tabular}

${ }^{a}$ 邻氨基苯甲酸与苯甲醛的物质的量比; ${ }^{b}$ 生成亚胺时, 加催化量的冰 醋酸.

\section{2 连有氨基侧链的喹唑啉酮衍生物的合成}

稠杂喹唑啉酮衍生物因其具有较大的共轭体系，易 于与 DNA 结合, 表现出显著的抗肿瘤活性, 如色胺 酮 ${ }^{[13]}$ 、骆驼宁碱 ${ }^{[14]}$ 等天然喹唑啉酮化合物. 因此, 在获 得含氨基酸酯链的喹唑啉-4-酮衍生物 5a 后, 我们将其 水解成酸, 拟用分子内傅-克(Friedel-Crafts)酰基化, 以 制备稠杂喹唑啉酮 7a (Scheme 3). 尝试了不同的强质子 酸如多聚磷酸(PPA)、浓硫酸、三氟醋酸等, 酸性条件下 脱水关环; 或将 $\mathbf{5 a}$ 在二氯亚砜作用下制备成酰氯，在路 易斯酸(Lewis acid)如四氯化钛、氯化锌等作用下傅-克 酰基化关环，没有得到预期的目标化合物 7a. 部分反 应, 如 PPA、四氯化钛催化或二氯亚砜直接为溶剂(不加 催化剂), 生成了羧甲基脱除的产物 8a，收率 28\% $30 \%$. 反应机理可能是: 羧基在氯化亚砜(或酸性条件) 作用下形成酰氯，羧甲基上的亚甲基更为活泼，易受到 $\mathrm{Cl}^{-}$的进攻, 进而脱除氯乙酰基得到 8a (Scheme 4). 这 与文献[15]报道一致. 同样条件下, 以 $\mathbf{5 b}$ 为原料得到化 合物 8b. 该方法可作为一个制备 C-2 位取代的喹唑啉4-酮衍生物的补充反应. 
<smiles>[R]C(C)c1ccc2c(=O)n3c(nc2c1)-c1ccccc1C(=O)C3</smiles>

$7 a$<smiles>[R]CCOc1nc([Al])nc2ccccc12</smiles>

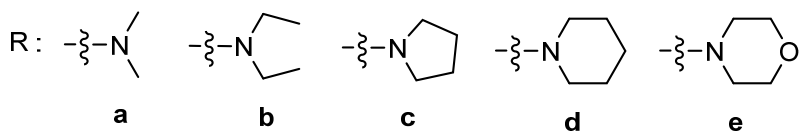

Reagents and conditions: (a) EtOH, $1 \mathrm{~mol} / \mathrm{L} \mathrm{NaOH}$, r.t., $5 \mathrm{~min}$; (b) $\mathrm{SOCl}_{2}$, reflux, $3 \mathrm{~h}$; (c) acetone, $\mathrm{K}_{2} \mathrm{CO}_{3}, \mathrm{RCH}_{2} \mathrm{CH}_{2} \mathrm{Cl}$, $\mathrm{KI}$, reflux, $3 \sim 4$

图式 3 化合物 9 的合成

Scheme 3 Synthesis of compound 9

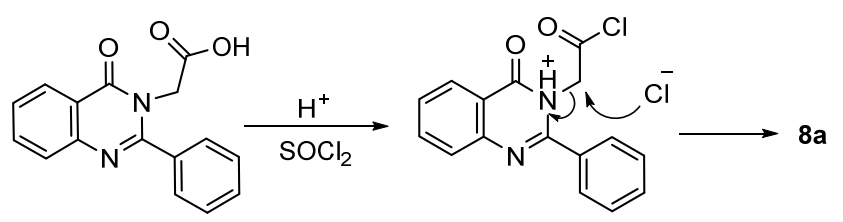

图式 4 化合物 $8 \mathrm{a}$ 的合成机理

Scheme 4 Possible mechanism for the synthesis of compound 8a

考虑到氨乙基侧链可以改善化合物的溶解性, 并增 强化合物与 DNA 的结合, 提高化合物的抗肿瘤活 性 ${ }^{[16,17]}$, 化合物 8 与氯乙基胺盐酸盐反应，以 $60 \%$ 90\%的产率制备了连有氨基侧链的喹唑啉衍生物 9 (Scheme 3). 反应伴随有少量 N-取代产物生成 ${ }^{[18]}$, 但难 以分离得到.

\section{2 化合物抗肿瘤活性评价}

对所合成的化合物进行了体外抑制人乳腺癌(MCF7)、白血病(K562)、肺癌(A549)、宫颈癌(hela)细胞活性 测试, 顺铂为阳性对照, 结果见表 2. 总体而言, 大部分 含香豆素的系列化合物具有较含苯环的系列化合物更 好的抗肿瘤细胞增殖活性, 这表明 C-2 为具有较苯环更 强抗肿瘤活性的香豆素 ${ }^{[19]}$, 或增加此处芳环的共轭平 面体系, 可能有助于提高化合物抗肿瘤活性. 氨基侧链 的不同使得化合物表现出一定的抗肿瘤细胞的选择性, 其中化合物 $9 b a$ 和 $9 b c$ 具有较好的抗 Hela 细胞增殖活 性, 而化合物 9bc 和 9bd 具有较好的抗 A549 细胞增殖 活性, 它们的 $\mathrm{IC}_{50}$ 值均低于 $10 \mu \mathrm{mol} / \mathrm{L}$, 且高于阳性对照 顺铂. 不含氨基侧链的化合物 $8 \mathrm{a}$ 和 $8 \mathrm{~b}$, 以及含吗啉侧
链的化合物 9ae 和 9be 均没有活性, 进一步表明氨基侧 链有利于化合物的抗肿瘤细胞增殖活性. 而链的长短以 及吗啉环对活性的影响还有待进一步研究. 以上构效关 系分析将有助于指导喹唑啉-4-酮类抗肿瘤化合物的结 构修饰。

表 2 化合物 8 和 9 的抗肿瘤活性

Table 2 Antitumor activities of compounds 8 and 9

\begin{tabular}{cllll}
\hline \multirow{2}{*}{ Compd. } & \multicolumn{4}{c}{ 抗肿瘤活性 $\left[\mathrm{IC}_{50} /\left(\mu \mathrm{mol} \cdot \mathrm{L}^{-1}\right)\right]$} \\
\cline { 2 - 5 } & MCF-7 & \multicolumn{1}{c}{$\mathrm{K} 562$} & \multicolumn{1}{c}{$\mathrm{A} 549$} & Hela \\
\hline $\mathbf{8 a}$ & $>50$ & $>50$ & $>50$ & $>50$ \\
$\mathbf{9 a a}$ & $>50$ & $>50$ & $>50$ & $>50$ \\
$\mathbf{9 a b}$ & $>50$ & $>50$ & $>50$ & $>50$ \\
$\mathbf{9 a c}$ & $>50$ & $>50$ & $>50$ & $>50$ \\
$\mathbf{9 a d}$ & $>50$ & $>50$ & $>50$ & $>50$ \\
$\mathbf{9 a e}$ & $>50$ & $>50$ & $>50$ & $>50$ \\
$\mathbf{8 b}$ & $>50$ & $>50$ & $>50$ & $>50$ \\
$\mathbf{9 b a}$ & $>50$ & $24.59 \pm 5.21$ & $>50$ & $8.16 \pm 0.78$ \\
$\mathbf{9 b b}$ & $>50$ & $43.25 \pm 7.60$ & $>50$ & $38.33 \pm 1.77$ \\
$\mathbf{9 b c}$ & $17.04 \pm 1.62$ & $12.49 \pm 1.58$ & $5.62 \pm 0.54$ & $7.47 \pm 0.17$ \\
$\mathbf{9 b d}$ & $29.87 \pm 5.74$ & $17.59 \pm 4.83$ & $9.54 \pm 1.83$ & $29.77 \pm 5.88$ \\
9be & $>50$ & $>50$ & $>50$ & $>50$ \\
顺铂 & $7.93 \pm 0.67$ & $3.00 \pm 0.71$ & $11.88 \pm 1.21$ & $12.07 \pm 1.28$ \\
\hline
\end{tabular}

\section{2 结论}

本文利用亚胺和亚胺烯酮的加成反应，以邻氨基苯 甲酸、芳醛和甘氨酸乙酯为原料, 一锅法构建了系列含 氨基酸链的 C-2 和 N-3 双取代的二氢喹唑啉-4-酩衍生物 4, 并芳构化得到喹唑啉-4-酮衍生物 5. 酸性条件下脱除 羧甲基，设计合成了连有氨基侧链的喹唑啉酮衍生物 $\mathbf{9}$, 并评价了化合物的抗肿瘤细胞增殖性. 其中化合物 9ba、9bc 和 9bd 具有良好的抗肿瘤活性，并表现出一定 的选择性. 构效关系分析表明, C-2 位香豆素(较大共轭 平面体系)取代以及氨基柔性侧链的引入将有利于化合 物的抗肿瘤细胞增殖活性.

\section{3 实验部分}

\section{1 仪器与试剂}

微波合成仪为美国 CEM 公司的 DISCOVER S-Class; 熔点由 $\mathrm{SGW}^{\circledR}$ X-4 显微熔点仪(温度计未校正) 测定; 核磁共振谱用 BRUKER AC-P600 (600 MHz)型核 磁共振仪测定, TMS 为内标; 质谱(ESI)用 Agilent G6300 离子阱液相质谱联用仪; 元素分析用 HERAEUS (CHNO, rapid)型元素分析仪; 光密度用 BioRad 3550 型 酶标仪测定; 层析用硅胶(200 300 目)为青岛海洋化工 厂产品. 本文所用其他试剂均为分析纯, 无水试剂均按 常规方法处理, 水为二次蒸馏水. 


\section{2 实验方法}

\section{2 .1 化合物 $4 \mathrm{a}$ 和 $4 \mathrm{~b}$ 的合成}

以化合物 $4 \mathbf{a}$ 的合成为例, 参照文献[9]方法. 将邻 氨基苯甲酸 $137 \mathrm{mg}(1.0 \mathrm{mmol})$ 溶于 $3 \mathrm{~mL}$ 无水苯中, 再 加入氯化亚砜 $595 \mathrm{mg}(5.0 \mathrm{mmol})$, 氮气保护下, 回流反 应 $3 \mathrm{~h}$. 反应结束后, 冷却至室温. 蒸除过量的氯化亚砜 和溶剂苯, 得到黄色油状物亚胺烯酮中间体 1 , 干燥备 用.

在 $50 \mathrm{~mL}$ 的圆底烧瓶中, 加入 $106 \mathrm{mg}(1.0 \mathrm{mmol})$ 苯 甲醛(2a)、210 mg (1.5 mmol) 甘氨酸乙酯盐酸盐、 $5 \mathrm{~mL}$ 无水苯以及 $2 \mathrm{~mL}(2.0 \mathrm{mmol}) \quad N, N^{\prime}$-二异丙基乙胺 (DIPEA), 氮气保护下室温搅拌. 以一滴冰醋酸为催化 剂, TLC 监测反应结束后, 得到中间体亚胺 $\mathbf{3 a}$, 备用.

将上述 $\mathbf{3 a}$ 的反应液倒入亚胺烯酮中间体 $\mathbf{1}$ 中, 室温 下剧烈摚拌 $8 \mathrm{~h}$, 簿层色谱(TLC)监测反应结束后, 加水 $(5 \mathrm{~mL} \times 2)$ 洗涤反应液. 有机相用无水硫酸镁干燥, 抽滤 浓缩, 所得粗产品用硅胶柱快速分离(石油醚/乙酸乙酯, $V: V=1: 1$ ), 再重结晶(石油醚/乙酸乙酯, $V: V=3:$ 1)得到 $170 \mathrm{mg}$ 化合物 4a. 按相同的方法, 以 7-香豆素醛 $\mathbf{2 b}$ 为原料, 得到化合物 $\mathbf{4 b}$.

2-[4-氧亚基-2-苯基-1,2-二氢喹唑啉-3(4H)-基]乙酸 乙酯(4a): 白色固体, 产率 $72.0 \%$. m.p. $155 \sim 159{ }^{\circ} \mathrm{C} ;{ }^{1} \mathrm{H}$ NMR (600 MHz, $\left.\mathrm{CDCl}_{3}\right) \delta: 7.98(\mathrm{~d}, J=7.8 \mathrm{~Hz}, 1 \mathrm{H}, \mathrm{Ar}-\mathrm{H})$, $7.52(\mathrm{t}, J=6.0 \mathrm{~Hz}, 2 \mathrm{H}, \mathrm{Ar}-\mathrm{H}), 7.41 \sim 7.44$ (m, 3H, Ar-H), $7.29 \sim 7.32(\mathrm{~m}, 1 \mathrm{H}, \mathrm{Ar}-\mathrm{H}), 6.89(\mathrm{t}, J=7.6 \mathrm{~Hz}, 1 \mathrm{H}, \mathrm{Ar}-\mathrm{H})$, 6.62 (d, J=7.8 Hz, 1H, Ar-H), 6.08 (s, 1H, CH), 4.65 (d, $\left.J=18.0 \mathrm{~Hz}, 1 \mathrm{H}, \mathrm{CH}_{2}\right), 4.13\left(\mathrm{t}, J=7.2 \mathrm{~Hz}, 2 \mathrm{H}, \mathrm{CH}_{2}\right), 3.27$ (d, $\left.J=17.4 \mathrm{~Hz}, 1 \mathrm{H}, \mathrm{CH}_{2}\right), 1.21\left(\mathrm{t}, J=7.2 \mathrm{~Hz}, 3 \mathrm{H}, \mathrm{CH}_{3}\right)$; MS (ESI) $m / z: 311.2\left([\mathrm{M}+\mathrm{H}]^{+}\right)$. Anal. calcd for $\mathrm{C}_{18} \mathrm{H}_{18} \mathrm{~N}_{2} \mathrm{O}_{3}$ : C 69.66, H 5.85, N 9.03; found $\mathrm{C} 69.74, \mathrm{H}$ 5.82, N 9.96 .

2-[2-(4-甲基-2-氧亚基- $2 H$-苯并二氢吡喃-7-基)-4氧亚基-1,2-二氢喹唑啉-3(4H)-基]乙酸乙酯(4b): 白色固 体, 产率 50.0\%. m.p. 232 233 ${ }^{\circ} \mathrm{C} ;{ }^{1} \mathrm{H}$ NMR (600 MHz, $\left.\mathrm{CDCl}_{3}\right) \delta: 7.93$ (d, $\left.J=7.8 \mathrm{~Hz}, 1 \mathrm{H}, \mathrm{Ar}-\mathrm{H}\right), 7.61$ (d, $J=8.2$ $\mathrm{Hz}, 1 \mathrm{H}, \mathrm{Ar}-\mathrm{H}), 7.45$ (d, $J=8.2 \mathrm{~Hz}, 1 \mathrm{H}, \mathrm{Ar}-\mathrm{H}), 7.40$ (s, 1H, Ar-H), 7.29 (t, $J=7.7 \mathrm{~Hz}, 1 \mathrm{H}, \mathrm{Ar}-\mathrm{H}), 6.88$ (t, $J=7.6 \mathrm{~Hz}$, $1 \mathrm{H}, \operatorname{Ar}-\mathrm{H}), 6.62$ (d, J=8.0 Hz, 1H, Ar-H), $6.31(\mathrm{~s}, 1 \mathrm{H}$, Ar-H), 6.09 (s, 1H, Ar-H), 4.74 (d, $J=17.6 \mathrm{~Hz}, 1 \mathrm{H}, \mathrm{CH}_{2}$ ), 4.58 (s, 1H, NH), 4.13 (dd, $\left.J=9.8,7.1 \mathrm{~Hz}, 2 \mathrm{H}, \mathrm{CH}_{2}\right), 3.29$ (d, $\left.J=17.6 \mathrm{~Hz}, 1 \mathrm{H}, \mathrm{CH}_{2}\right), 2.42\left(\mathrm{~s}, 3 \mathrm{H}, \mathrm{CH}_{3}\right), 1.21(\mathrm{t}, J=$ $\left.7.1 \mathrm{~Hz}, 3 \mathrm{H}, \mathrm{CH}_{3}\right)$; MS (ESI) $\mathrm{m} / z: 415.4\left([\mathrm{M}+\mathrm{Na}]^{+}\right)$. Anal. calcd for $\mathrm{C}_{22} \mathrm{H}_{20} \mathrm{~N}_{2} \mathrm{O}_{5}$ : C 67.34, $\mathrm{H} \mathrm{5.14,} \mathrm{N} 7.14$; found $\mathrm{C}$ 67.36, H 5.21, N 7.10.

\section{2 .2 化合物 $5 \mathbf{a}$ 和 $\mathbf{5 b}$ 的合成}

$50 \mathrm{~mL}$ 圆底烧瓶中加入 $310 \mathrm{mg}(1.0 \mathrm{mmol})$ 化合物 4a、395 mg (2.5 mmol)高锰酸钾、 $5 \mathrm{~mL}$ 无水丙酮, 加热 回流, TLC 监测反应. $3 \mathrm{~h}$ 后反应完全, 反应液冷却至室 温后, 抽滤除去不溶物, 母液减压除去溶剂, 得到的粗 产品经乙酸乙酯重结晶得到 $295 \mathrm{mg}$ 化合物 5a. 按相同 的方法，以化合物 $\mathbf{4 b}$ 为原料，得到化合物 $\mathbf{5 b}$.

2-[4-氧亚基-2-苯基喹唑啉-3(4H)-基]乙酸乙酯(5a): 白色固体，产率 96.0\%. m.p. 125 $129{ }^{\circ} \mathrm{C} ;{ }^{1} \mathrm{H}$ NMR $(600$ $\left.\mathrm{MHz}, \mathrm{CDCl}_{3}\right) \delta: 8.35(\mathrm{~d}, J=8.4 \mathrm{~Hz}, 1 \mathrm{H}, \mathrm{Ar}-\mathrm{H}), 7.79 \sim$ $7.83(\mathrm{~m}, 2 \mathrm{H}, \mathrm{Ar}-\mathrm{H}), 7.51 \sim 7.59(\mathrm{~m}, 6 \mathrm{H}, \mathrm{Ar}-\mathrm{H}), 4.67(\mathrm{~s}$, $\left.2 \mathrm{H}, \mathrm{CH}_{2}\right), 4.24\left(\mathrm{q}, J=7.2 \mathrm{~Hz}, 2 \mathrm{H}, \mathrm{CH}_{2}\right), 1.27(\mathrm{t}, J=7.2$ $\left.\mathrm{Hz}, 3 \mathrm{H}, \mathrm{CH}_{3}\right)$; MS (ESI) $m / z: 309.2\left([\mathrm{M}+\mathrm{H}]^{+}\right)$. Anal. calcd for $\mathrm{C}_{18} \mathrm{H}_{16} \mathrm{~N}_{2} \mathrm{O}_{3}$ : C 70.12, H 5.23, N 9.09; found $\mathrm{C}$ 70.21, H 5.26, N 9.03 .

2-[2-(4-甲基-2-氧亚基- $2 H$-苯并二氢吡喃-7-基)-4氧亚基喹唑啉-3(4H)-基]乙酸乙酯(5b): 白色固体, 产率 96.0\%. m.p. 189 $191{ }^{\circ} \mathrm{C} ;{ }^{1} \mathrm{H}$ NMR (600 MHz, $\left.\mathrm{CDCl}_{3}\right) \delta$ : $8.35(\mathrm{t}, J=6.6 \mathrm{~Hz}, 1 \mathrm{H}, \mathrm{Ar}-\mathrm{H}), 7.85 \sim 7.83(\mathrm{~m}, 1 \mathrm{H}, \mathrm{Ar}-\mathrm{H})$, $7.79 \sim 7.75(\mathrm{~m}, 2 \mathrm{H}, \mathrm{Ar}-\mathrm{H}), 7.59 \sim 7.54(\mathrm{~m}, 3 \mathrm{H}, \mathrm{Ar}-\mathrm{H}), 6.42$ (s, 1H, NH), $4.66\left(\mathrm{~s}, 2 \mathrm{H}, \mathrm{CH}_{2}\right), 4.37$ (dd, $J=7.2 \mathrm{~Hz}, 2 \mathrm{H}$, $\left.\mathrm{CH}_{2}\right), 2.52$ (s, 3H, $\left.\mathrm{CH}_{3}\right), 1.29$ (t, $\left.J=7.2 \mathrm{~Hz}, 3 \mathrm{H}, \mathrm{CH}_{3}\right)$; MS (ESI) $m / z: 413.4\left([\mathrm{M}+\mathrm{Na}]^{+}\right)$. Anal. calcd for $\mathrm{C}_{22} \mathrm{H}_{18} \mathrm{~N}_{2} \mathrm{O}_{5}$ : C 67.69, H 4.65, N 7.18; found C 67.78, H 4.72, N 7.20.

\section{2 .3 化合物 $6 \mathrm{a}$ 和 $6 \mathrm{~b}$ 的合成}

$50 \mathrm{~mL}$ 圆底烧瓶中加入 $616 \mathrm{mg}(2.0 \mathrm{mmol})$ 化合物 5a, 加入 $5.0 \mathrm{~mL}$ 无水乙醇, 室温摚拌下加入 $1 \mathrm{~mol} / \mathrm{L}$ $\mathrm{NaOH}$ 溶液 $1 \mathrm{~mL}$, 继续搅拌 $5 \mathrm{~min}$, 反应液变澄清, TLC 监测反应完全. 反应液用 $1 \mathrm{~mol} / \mathrm{L}$ 盐酸酸化至 $\mathrm{pH}<1$, 静 置抽滤, 滤饼用乙酸乙酯重结晶得到 $550 \mathrm{mg}$ 化合物 6 . 按相同的方法，以化合物 $\mathbf{5 b}$ 为原料，得到化合物 $\mathbf{6 b}$.

2-[4-氧亚基-2-苯基喹唑啉-3(4H)-基]乙酸(6a)：白 色固体, 产率 98.0\%. m.p. 233 237 ${ }^{\circ} \mathrm{C} ;{ }^{1} \mathrm{H}$ NMR $(600$ $\left.\mathrm{MHz}, \mathrm{CD}_{3} \mathrm{OD}\right) \delta: 8.28(\mathrm{~d}, J=7.2 \mathrm{~Hz}, 1 \mathrm{H}, \mathrm{Ar}-\mathrm{H}), 7.90(\mathrm{t}$, $J=7.2 \mathrm{~Hz}, 1 \mathrm{H}, \operatorname{Ar}-\mathrm{H}), 7.73(\mathrm{t}, J=8.4 \mathrm{~Hz}, 1 \mathrm{H}, \mathrm{Ar}-\mathrm{H})$, $7.63 \sim 7.57(\mathrm{~m}, 6 \mathrm{H}, \mathrm{Ar}-\mathrm{H}), 4.66\left(\mathrm{~s}, 2 \mathrm{H}, \mathrm{CH}_{2}\right)$; MS (ESI) $m / z: 281.2\left([\mathrm{M}+\mathrm{H}]^{+}\right)$. Anal. calcd for $\mathrm{C}_{16} \mathrm{H}_{12} \mathrm{~N}_{2} \mathrm{O}_{3}: \mathrm{C}$ 68.56, H 4.32, N 9.99; found C 68.54, H 4.36, N 10.02.

2-[2-(4-甲基-2-氧亚基- $2 H$-苯并二氢吡喃-7-基)-4氧亚基喹唑啉-3(4H)-基]乙酸(6b): 白色固体，产率 96.0\%. m.p. 266 267 ${ }^{\circ} \mathrm{C} ;{ }^{1} \mathrm{H}$ NMR (600 MHz, $\left.\mathrm{CD}_{3} \mathrm{OD}\right)$ $\delta: 8.30(\mathrm{~d}, J=8.4 \mathrm{~Hz}, 1 \mathrm{H}, \mathrm{Ar}-\mathrm{H}), 7.99$ (d, $J=7.8 \mathrm{~Hz}, 1 \mathrm{H}$, Ar-H), 7.91 (t, $J=7.8 \mathrm{~Hz}, 1 \mathrm{H}, \operatorname{Ar}-\mathrm{H}), 7.76$ (d, $J=8.4 \mathrm{~Hz}$, $1 \mathrm{H}, \mathrm{Ar}-\mathrm{H}), 7.66 \sim 7.61(\mathrm{~m}, 3 \mathrm{H}, \mathrm{Ar}-\mathrm{H}), 6.48(\mathrm{~s}, 1 \mathrm{H}, \mathrm{NH})$, 4.70 (s, $2 \mathrm{H} \mathrm{CH}_{2}$ ), 2.56 (s, 3H, $\mathrm{CH}_{3}$ ); MS (ESI) $m / z: 363.3$ 
$\left([\mathrm{M}+\mathrm{H}]^{+}\right)$. Anal. calcd for $\mathrm{C}_{20} \mathrm{H}_{14} \mathrm{~N}_{2} \mathrm{O}_{5}$ : C 66.30, H 3.89, N 7.73; found C 66.35, H 3.92, N 7.74.

\section{2 .4 化合物 $8 \mathrm{a}$ 和 $8 \mathrm{~b}$ 的合成}

$50 \mathrm{~mL}$ 圆底烧瓶中加入 $200 \mathrm{mg}(0.7 \mathrm{mmol})$ 化合物 6a, 加入 $5.0 \mathrm{~mL}$ 氯化亚砜, 回流反应 $3 \mathrm{~h}$, 反应结束后, 用氮气吹出氯化亚砜, 残留物用甲醇重结晶. 所得粗品 经硅胶柱(石油醚/乙酸乙酯, $V: V=3: 1$ )分离得到 63 $\mathrm{mg}$ 化合物 8a. 按相同的方法, 以化合物 $\mathbf{6 b}$ 为原料, 得 到化合物 8b.

2- 苯基喹唑啉-3(4H)-酮 (8a): 白色固体, 产率 28.0\%. m.p. $252 \sim 253{ }^{\circ} \mathrm{C} ;{ }^{1} \mathrm{H}$ NMR (600 MHz, $\left.\mathrm{CDCl}_{3}\right)$ : $\delta: 11.71(\mathrm{~s}, 1 \mathrm{H}, \mathrm{OH}), 8.33(\mathrm{~d}, J=6.6 \mathrm{~Hz}, 1 \mathrm{H}, \mathrm{Ar}-\mathrm{H})$, $8.27 \sim 8.25$ (m, 2H, Ar-H), 7.85 (d, J=7.8 Hz, 1H, Ar-H), $7.81(\mathrm{t}, J=7.8 \mathrm{~Hz}, 1 \mathrm{H}, \mathrm{Ar}-\mathrm{H}), 7.59 \sim 7.58(\mathrm{~m}, 3 \mathrm{H}, \mathrm{Ar}-\mathrm{H})$, 7.51 (t, $J=7.8 \mathrm{~Hz}, 1 \mathrm{H}, \mathrm{Ar}-\mathrm{H})$; MS (ESI) $m / z: 361.2$ ([M+ $\mathrm{K}]^{+}$). Anal. calcd for $\mathrm{C}_{14} \mathrm{H}_{10} \mathrm{~N}_{2} \mathrm{O}: \mathrm{C} 75.66, \mathrm{H} 4.54, \mathrm{~N}$ 12.60; found C 75.73, H 4.57, N 12.56 .

2-(4-甲基-2-氧亚基- $2 H$-苯并二氢吡喃-7-基)-喹唑 啉-3(4H)-酮 (8b): 白色固体, 产率 36.7\%. m.p. 263 $265{ }^{\circ} \mathrm{C} ;{ }^{1} \mathrm{H}$ NMR $\left(600 \mathrm{MHz}\right.$, Pyridine- $\left.d_{5}\right) \delta: 8.59$ (d, $J=$ $7.8 \mathrm{~Hz}, 1 \mathrm{H}, \mathrm{Ar}-\mathrm{H}), 8.45$ (s, $1 \mathrm{H}, \mathrm{Ar}-\mathrm{H}), 8.39$ (t, $J=7.2 \mathrm{~Hz}$, $1 \mathrm{H}, \mathrm{Ar}-\mathrm{H}), 8.03$ (d, $J=7.8 \mathrm{~Hz}, 1 \mathrm{H}, \mathrm{Ar}-\mathrm{H}), 7.81$ (d, $J=8.4$ $\mathrm{Hz}, 2 \mathrm{H}, \mathrm{Ar}-\mathrm{H}), 7.51$ (t, J=7.8 Hz, 1H, Ar-H), 6.42 (s, 1H, $\mathrm{CH}), 2.26$ (s, $3 \mathrm{H}, \mathrm{CH}_{3}$ ); $\mathrm{MS}$ (ESI) $m / z: 305.3\left([\mathrm{M}+\mathrm{H}]^{+}\right.$). Anal. calcd for $\mathrm{C}_{18} \mathrm{H}_{12} \mathrm{~N}_{2} \mathrm{O}_{3}$ : C 71.05, H 3.97, N 9.21; found $\mathrm{C} 71.12, \mathrm{H} 4.04, \mathrm{~N} 9.19$.

\section{2 .5 化合物 9aa 9ae 和 9ba 9be 的合成}

以化合物 9aa 的合成为例: $50 \mathrm{~mL}$ 圆底烧瓶中加入 $200 \mathrm{mg}(0.76 \mathrm{mmol})$ 化合物 $\mathbf{8 a} 、 210 \mathrm{mg}(1.5 \mathrm{mmol})$ 无水碳 酸钾, 再加入 $10 \mathrm{~mL}$ 新处理的丙酮, 回流反应 $30 \mathrm{~min}$ 后 加入 $164 \mathrm{mg}(1.1 \mathrm{mmol}) 2$-氯 $-N, N$-二甲基乙胺盐酸盐和 催化量碘化钾, 继续回流反应 $4 \mathrm{~h}$, 反应结束后抽滤除 去碳酸钾, 母液浓缩后经柱色谱分离(石油醚/乙酸乙酯, $V: V=3: 1$ )得到 $193 \mathrm{mg}$ 化合物 9aa. 按相同的方法, 以不同的氯代乙胺盐酸盐为原料, 得到化合物 $\mathbf{9 a b} \sim$ $9 \mathrm{ae}$ 和 9ba $\sim 9 \mathrm{be}$ ( $8 \mathrm{~b}$ 为原料).

$\mathrm{N}, \mathrm{N}$-二甲基-2-[(2-苯基喹唑啉-4-基)氧基]乙胺 (9aa): 黄色油状物, 产率 46.9\%. ${ }^{1} \mathrm{H}$ NMR $(600 \mathrm{MHz}$, $\left.\mathrm{CDCl}_{3}\right) \delta: 8.62 \sim 8.59(\mathrm{~m}, 2 \mathrm{H}, \mathrm{Ar}-\mathrm{H}), 8.20(\mathrm{~d}, J=8.1 \mathrm{~Hz}$, 1H, Ar-H), 8.01 (d, $J=8.3 \mathrm{~Hz}, 1 \mathrm{H}, \mathrm{Ar}-\mathrm{H}), 7.83$ (dd, $J=$ 11.1, $4.2 \mathrm{~Hz}, 1 \mathrm{H}, \mathrm{Ar}-\mathrm{H}), 7.55 \sim 7.51$ (m, 4H, Ar-H), 4.89 (t, $\left.J=5.8 \mathrm{~Hz}, 2 \mathrm{H}, \mathrm{CH}_{2}\right), 2.98\left(\mathrm{t}, J=5.8 \mathrm{~Hz}, 2 \mathrm{H}, \mathrm{CH}_{2}\right), 2.47$ (s, $\left.6 \mathrm{H}, \mathrm{CH}_{3}\right)$; MS (ESI) $m / z: 316.4\left([\mathrm{M}+\mathrm{Na}]^{+}\right)$. Anal. calcd for $\mathrm{C}_{18} \mathrm{H}_{19} \mathrm{~N}_{3} \mathrm{O}$ : C 73.69, H 6.53, N 14.32; found $\mathrm{C}$ 73.72, H 6.58, N 14.33.
$\mathrm{N}, \mathrm{N}$-二乙基-2-[(2-苯基喹唑啉-4-基) 氧基] 乙胺 (9ab): 黄色油状物, 产率 45.3\%. ${ }^{1} \mathrm{H}$ NMR $(600 \mathrm{MHz}$, $\left.\mathrm{CDCl}_{3}\right) \delta: 8.61$ (d, $\left.J=7.2 \mathrm{~Hz}, 2 \mathrm{H}, \mathrm{Ar}-\mathrm{H}\right), 8.18$ (d, $J=7.8$ $\mathrm{Hz}, 1 \mathrm{H}, \mathrm{Ar}-\mathrm{H}), 8.01$ (d, $J=8.4 \mathrm{~Hz}, 1 \mathrm{H}, \mathrm{Ar}-\mathrm{H}), 7.83$ (t, $J=$ $7.2 \mathrm{~Hz}, 1 \mathrm{H}, \mathrm{Ar}-\mathrm{H}), 7.54 \sim 7.51$ (m, 4H, Ar-H), 4.84 (t, $J=$ $6.0 \mathrm{~Hz}, 2 \mathrm{H}, \mathrm{CH}_{2}$ ), 3.09 (t, $J=6.0 \mathrm{~Hz}, 2 \mathrm{H}, \mathrm{CH}_{2}$ ), 2.76 (q, $\left.J=7.2 \mathrm{~Hz}, 2 \mathrm{H}, \mathrm{CH}_{2}\right), 1.15\left(\mathrm{t}, J=7.2 \mathrm{~Hz}, 6 \mathrm{H}, \mathrm{CH}_{3}\right) ; \mathrm{MS}$ (ESI) $m / z: 344.2\left([\mathrm{M}+\mathrm{Na}]^{+}\right)$. Anal. calcd for $\mathrm{C}_{20} \mathrm{H}_{23} \mathrm{~N}_{3} \mathrm{O}$ : C 74.74, H 7.21, N 13.07; found C 74.78, H 7.19, N 13.06.

2-苯基-4-[2-(四氢吡咯-1-基)乙氧基]喹唑啉(9ac): 黄色油状物, 产率 $56.7 \% .{ }^{1} \mathrm{H}$ NMR $\left(600 \mathrm{MHz}, \mathrm{CD}_{3} \mathrm{OD}\right)$ $\delta: 8.55 \sim 8.53(\mathrm{~m}, 2 \mathrm{H}, \operatorname{Ar}-\mathrm{H}), 8.27(\mathrm{~d}, J=8.4 \mathrm{~Hz}, 1 \mathrm{H}$, Ar-H), 7.89 (d, $J=8.4 \mathrm{~Hz}, 1 \mathrm{H}, \mathrm{Ar}-\mathrm{H}), 7.91$ (t, $J=8.4 \mathrm{~Hz}$, $1 \mathrm{H}, \mathrm{Ar}-\mathrm{H}), 7.63 \sim 7.61(\mathrm{~m}, 1 \mathrm{H}, \mathrm{Ar}-\mathrm{H}), 7.53 \sim 7.52(\mathrm{~m}, 3 \mathrm{H}$, Ar-H), 4.93 (t, $\left.J=5.4 \mathrm{~Hz}, 2 \mathrm{H}, \mathrm{CH}_{2}\right), 3.18$ (t, $J=5.4 \mathrm{~Hz}$, 2H, $\mathrm{CH}_{2}$ ), 2.83 (brs, 4H, $\mathrm{CH}_{2}$ ), 1.89 (brs, $4 \mathrm{H}, \mathrm{CH}_{2}$ ); MS (ESI) $m / z$ : $320.3\left([\mathrm{M}+\mathrm{H}]^{+}\right)$. Anal. calcd for $\mathrm{C}_{20} \mathrm{H}_{23} \mathrm{~N}_{3} \mathrm{O}: \mathrm{C}$ 75.21, H 6.63, N 13.16; found C 75.24, H 6.65, N 13.09.

2-苯基-4-[2-(哌啶-1-基)乙氧基]喹唑啉(9ad)：黄色 油状物, 产率 90.2\%. ${ }^{1} \mathrm{H}$ NMR (600 MHz, $\left.\mathrm{CDCl}_{3}\right) \delta: 8.61$ (d, $J=6.6 \mathrm{~Hz}, 2 \mathrm{H}, \mathrm{Ar}-\mathrm{H}), 8.18(\mathrm{~d}, J=7.2 \mathrm{~Hz}, 1 \mathrm{H}, \mathrm{Ar}-\mathrm{H})$, $8.01(\mathrm{~d}, J=8.4 \mathrm{~Hz}, 1 \mathrm{H}$, Ar-H), $7.84(\mathrm{t}, J=7.2 \mathrm{~Hz}, 1 \mathrm{H}$, Ar-H), $7.54 \sim 7.52$ (m, 4H, Ar-H), 4.89 (t, $J=6.0 \mathrm{~Hz}, 2 \mathrm{H}$, $\mathrm{CH}_{2}$ ), 2.98 (t, $J=6.0 \mathrm{~Hz}, 2 \mathrm{H}, \mathrm{CH}_{2}$ ), 2.64 (brs, $4 \mathrm{H}, \mathrm{CH}_{2}$ ), $1.68 \sim 1.64$ (m, 4H, $\mathrm{CH}_{2}$ ), 1.49 (brs, $2 \mathrm{H}, \mathrm{CH}_{2}$ ); MS (ESI) $m / z: 356.2\left([\mathrm{M}+\mathrm{Na}]^{+}\right)$. Anal. calcd for $\mathrm{C}_{21} \mathrm{H}_{23} \mathrm{~N}_{3} \mathrm{O}$ : C 75.65, H 6.95, N 12.60; found C 75.71, H 6.88, N 13.69.

4-\{2-[(2-苯基喹唑啉-4-基)氧基]乙基\}吗啉(9ae): 黄色油状物, 产率 92.4\%. ${ }^{1} \mathrm{H}$ NMR $\left(600 \mathrm{MHz}, \mathrm{CDCl}_{3}\right) \delta$ : $8.59(\mathrm{dd}, J=2.4,6.6 \mathrm{~Hz}, 2 \mathrm{H}, \mathrm{Ar}-\mathrm{H}), 8.18(\mathrm{~d}, J=6.6 \mathrm{~Hz}$, $1 \mathrm{H}, \mathrm{Ar}-\mathrm{H}), 8.02$ (d, $J=8.4 \mathrm{~Hz}, 1 \mathrm{H}, \mathrm{Ar}-\mathrm{H}), 7.85$ (dt, $J=1.2$, $5.4 \mathrm{~Hz}, 1 \mathrm{H}, \mathrm{Ar}-\mathrm{H}), 7.56 \sim 7.51(\mathrm{~m}, 4 \mathrm{H}, \mathrm{Ar}-\mathrm{H}), 4.90(\mathrm{t}, J=$ $\left.6.0 \mathrm{~Hz}, 2 \mathrm{H}, \mathrm{CH}_{2}\right), 3.77$ (t, $\left.J=4.8 \mathrm{~Hz}, 4 \mathrm{H}, \mathrm{CH}_{2}\right), 3.00$ (t, $J=6.0 \mathrm{~Hz}, 2 \mathrm{H}, \mathrm{CH}_{2}$ ), 2.69 (brs, $4 \mathrm{H}, \mathrm{CH}_{2}$ ); MS (ESI) $\mathrm{m} / z$ : $336.4\left([\mathrm{M}+\mathrm{H}]^{+}\right)$. Anal. calcd for $\mathrm{C}_{20} \mathrm{H}_{21} \mathrm{~N}_{3} \mathrm{O}_{2}: \mathrm{C} 71.62, \mathrm{H}$ 6.31, N 12.53; found C 71.65, H 6.34, N 12.58 .

7-\{4-[2-(二甲基氨基)乙氧基]喹唑啉-2-基 $\}$-4-甲基$2 H$-苯并二氢吡喃-2-酮(9ba): 白色固体, 产率 $54.3 \%$. m.p. $128 \sim 129{ }^{\circ} \mathrm{C} ;{ }^{1} \mathrm{H}$ NMR (600 MHz, $\left.\mathrm{CDCl}_{3}\right) \delta: 8.52$ (d, $J=6.6 \mathrm{~Hz}, 2 \mathrm{H}, \mathrm{Ar}-\mathrm{H}), 8.19$ (d, $J=8.4 \mathrm{~Hz}, 1 \mathrm{H}, \mathrm{Ar}-\mathrm{H})$, 7.99 (d, $J=8.4 \mathrm{~Hz}, 1 \mathrm{H}, \mathrm{Ar}-\mathrm{H}), 7.84(\mathrm{t}, J=7.2 \mathrm{~Hz}, 1 \mathrm{H}$, Ar-H), 7.69 (d, $J=8.4 \mathrm{~Hz}, 1 \mathrm{H}, \mathrm{Ar}-\mathrm{H}), 7.55(\mathrm{t}, J=7.2 \mathrm{~Hz}$, $1 \mathrm{H}, \mathrm{Ar}-\mathrm{H}), 6.33$ (s, $1 \mathrm{H}, \mathrm{CH}), 4.84$ (t, $J=5.4 \mathrm{~Hz}, 2 \mathrm{H}, \mathrm{CH}_{2}$ ), $2.94\left(\mathrm{t}, J=5.4 \mathrm{~Hz}, 2 \mathrm{H}, \mathrm{CH}_{2}\right), 2.49\left(\mathrm{~s}, 3 \mathrm{H}, \mathrm{CH}_{3}\right), 2.43(\mathrm{~s}$, $\left.6 \mathrm{H}, \mathrm{CH}_{3}\right) ;{ }^{13} \mathrm{C}$ NMR $\left(150 \mathrm{MHz}, \mathrm{CDCl}_{3}\right) \delta: 166.8,160.9$, 
158.1, 153.7, 152.1, 151.71, 141.7, 133.8, 128.1, 127.1, 124.5, 123.9, 123.7, 121.4, 116.9, 115.6, 115.5, 65.2, 57.8, 46.0, 18.7; MS (ESI) $m / z: 398.4\left([\mathrm{M}+\mathrm{Na}]^{+}\right)$. Anal. calcd for $\mathrm{C}_{22} \mathrm{H}_{21} \mathrm{~N}_{3} \mathrm{O}_{3}$ : C 70.38, H 5.64, N 11.19; found C 70.45, $\mathrm{H}$ 5.60, N 11.28.

7-\{4-[2-(二乙基氨基)乙氧基]喹唑啉-2-基\}-4-甲基$2 H$-苯并二氢吡喃-2-酮(9bb): 白色固体, 产率 $78.4 \%$. m.p. $126 \sim 127{ }^{\circ} \mathrm{C} ;{ }^{1} \mathrm{H}$ NMR $\left(600 \mathrm{MHz}, \mathrm{CDCl}_{3}\right) \delta: 8.55(\mathrm{~d}$, $J=6.6 \mathrm{~Hz}, 2 \mathrm{H}, \mathrm{Ar}-\mathrm{H}), 8.19$ (d, $J=7.8 \mathrm{~Hz}, 1 \mathrm{H}, \mathrm{Ar}-\mathrm{H}), 8.02$ (d, $J=8.4 \mathrm{~Hz}, 1 \mathrm{H}, \mathrm{Ar}-\mathrm{H}), 7.87$ (t, $J=7.2 \mathrm{~Hz}, 1 \mathrm{H}, \mathrm{Ar}-\mathrm{H}$ ), $7.72(\mathrm{~d}, J=8.4 \mathrm{~Hz}, 1 \mathrm{H}$, Ar-H), $7.58(\mathrm{t}, J=7.8 \mathrm{~Hz}, 1 \mathrm{H}$, Ar-H), $6.36(\mathrm{~s}, 1 \mathrm{H}, \mathrm{CH}), 4.88\left(\mathrm{t}, J=6.0 \mathrm{~Hz}, 2 \mathrm{H}, \mathrm{CH}_{2}\right)$, 3.16 (t, $\left.J=6.6 \mathrm{~Hz}, 2 \mathrm{H}, \mathrm{CH}_{2}\right), 2.82(\mathrm{~d}, J=7.2 \mathrm{~Hz}, 4 \mathrm{H}$, $\left.\mathrm{CH}_{2}\right), 2.51$ (s, 3H, $\left.\mathrm{CH}_{3}\right), 1.19$ (s, $\left.6 \mathrm{H}, \mathrm{CH}_{3}\right) ;{ }^{13} \mathrm{C} \mathrm{NMR}(150$ $\left.\mathrm{MHz}, \mathrm{CDCl}_{3}\right) \delta: 166.8,160.8,158.2,153.8,151.9,151.8$, $141.7,133.8,128.2,127.1,124.4,123.9,123.5,121.4$, 116.9, 115.7, 115.5, 51.0, 47.9, 18.6, 11.7; MS (ESI) $\mathrm{m} / \mathrm{z}$ : $426.2\left([\mathrm{M}+\mathrm{Na}]^{+}\right)$. Anal. calcd for $\mathrm{C}_{24} \mathrm{H}_{25} \mathrm{~N}_{3} \mathrm{O}_{3}: \mathrm{C} 71.44, \mathrm{H}$ 6.25, N 10.41; found C 71.46, H 6.24, N 10.42.

4-甲基-7-\{4-[2-(四氢吡咯-1-基)乙氧基]喹唑啉-2基 $\}$ - $2 \mathrm{H}$ - 苯并二氢吡喃-2-酮 (9bc): 白色固体, 产率 $80.2 \%$. m.p. $121 \sim 122{ }^{\circ} \mathrm{C} ;{ }^{1} \mathrm{H}$ NMR (600 MHz, $\left.\mathrm{CDCl}_{3}\right) \delta$ : $8.52(\mathrm{dd}, J=3.6,1.2 \mathrm{~Hz}, 2 \mathrm{H}, \mathrm{Ar}-\mathrm{H}), 8.19$ (d, $J=8.4 \mathrm{~Hz}$, $1 \mathrm{H}, \mathrm{Ar}-\mathrm{H}), 8.01$ (d, $J=8.4 \mathrm{~Hz}, 1 \mathrm{H}, \mathrm{Ar}-\mathrm{H}), 7.85$ (t, $J=7.2$ $\mathrm{Hz}, 1 \mathrm{H}, \mathrm{Ar}-\mathrm{H}), 7.68$ (d, $J=8.4 \mathrm{~Hz}, 1 \mathrm{H}, \mathrm{Ar}-\mathrm{H}), 7.56$ (t, $J=$ $7.2 \mathrm{~Hz}, 1 \mathrm{H}, \mathrm{Ar}-\mathrm{H}), 6.33(\mathrm{~s}, 1 \mathrm{H}, \mathrm{CH}), 4.89$ (t, $J=6.0 \mathrm{~Hz}$, $2 \mathrm{H}, \mathrm{CH}_{2}$ ), 3.13 (t, $\left.J=6.0 \mathrm{~Hz}, 2 \mathrm{H}, \mathrm{CH}_{2}\right), 2.77$ (s, 4H, $\mathrm{CH}_{2}$ ), 2.49 (s, $\left.3 \mathrm{H}, \mathrm{CH}_{3}\right), 1.87$ (s, $\left.4 \mathrm{H}, \mathrm{CH}_{2}\right) ;{ }^{13} \mathrm{C}$ NMR $(150 \mathrm{MHz}$, $\left.\mathrm{CDCl}_{3}\right) \delta: 166.6,160.8,153.6,152.0,151.7,141.6,133.7$, $128.1,127.0,124.4,123.9,123.6,121.3,116.8,115.6$, 115.5, 66.1, 54.8, 54.4, 23.6, 18.6; MS (ESI) $\mathrm{m} / z$ : 464.5 $\left([\mathrm{M}+\mathrm{Na}]^{+}\right)$. Anal. calcd for $\mathrm{C}_{24} \mathrm{H}_{23} \mathrm{~N}_{3} \mathrm{O}_{3}: \mathrm{C} 71.80$, H 5.77, N 10.47; found C 71.77, H 5.81, N 10.49 .

4- 甲基-7-\{4-[2-(哌啶-1-基) 乙氧基]喹唑啉-2基 \}- $2 \mathrm{H}$ - 苯并二氢吡喃-2-酮 (9bd): 白色固体, 产率 $81.8 \%$. m.p. $133 \sim 134{ }^{\circ} \mathrm{C} ;{ }^{1} \mathrm{H}$ NMR (600 MHz, $\left.\mathrm{CDCl}_{3}\right) \delta$ : 8.57 (d, $J=10.2 \mathrm{~Hz}, 2 \mathrm{H}, \mathrm{Ar}-\mathrm{H}), 8.21$ (d, $J=8.4 \mathrm{~Hz}, 1 \mathrm{H}$, Ar-H), 8.03 (d, $J=8.4 \mathrm{~Hz}, 1 \mathrm{H}, \mathrm{Ar}-\mathrm{H}), 7.87$ (t, $J=7.2 \mathrm{~Hz}$, $1 \mathrm{H}, \mathrm{Ar}-\mathrm{H}), 7.73$ (d, $J=7.8 \mathrm{~Hz}, 1 \mathrm{H}, \mathrm{Ar}-\mathrm{H}), 7.58$ (t, $J=7.2$ $\mathrm{Hz}, 1 \mathrm{H}, \mathrm{Ar}-\mathrm{H}), 6.37$ (s, 1H, CH), 4.90 (t, $J=6.0 \mathrm{~Hz}, 2 \mathrm{H}$, $\mathrm{CH}_{2}$ ), 3.02 (t, $J=6.0 \mathrm{~Hz}, 2 \mathrm{H}, \mathrm{CH}_{2}$ ), 2.67 (brs, $4 \mathrm{H}, \mathrm{CH}_{2}$ ), 2.52 (s, $\left.3 \mathrm{H}, \mathrm{CH}_{3}\right), 1.68$ (m, 4H, $\left.\mathrm{CH}_{2}\right), 1.51$ (brs, $2 \mathrm{H}, \mathrm{CH}_{2}$ ); ${ }^{13} \mathrm{C}$ NMR $\left(150 \mathrm{MHz}, \mathrm{CDCl}_{3}\right) \delta: 166.7,160.8,158.1,153.7$, $152.0,151.7,141.7,133.7,128.1,127.0,124.4,123.9$, 123.6, 121.3, 116.9, 115.6, 115.5, 64.9, 57.4, 54.9, 25.88,
24.1, 18.7; MS (ESI) $m / z: 438.5\left([\mathrm{M}+\mathrm{Na}]^{+}\right)$. Anal. calcd for $\mathrm{C}_{25} \mathrm{H}_{25} \mathrm{~N}_{3} \mathrm{O}_{3}$ : C 72.27, $\mathrm{H}$ 6.06, N 10.11; found $\mathrm{C} 72.20$, H 5.98, N 10.03 .

4-甲基-7-[4-(2-吗啉乙氧基)喹唑啉-2-基]- $2 \mathrm{H}$-苯并 二氢吡喃-2-酩(9be): 白色固体, 产率 61.9\%. m.p. 143 $144{ }^{\circ} \mathrm{C} ;{ }^{1} \mathrm{H}$ NMR $\left(600 \mathrm{MHz}, \mathrm{CDCl}_{3}\right) \delta: 8.54$ (d, $J=7.2$ $\mathrm{Hz}, 2 \mathrm{H}, \mathrm{Ar}-\mathrm{H}$ ), 8.18 (d, $J=8.4 \mathrm{~Hz}, 1 \mathrm{H}, \mathrm{Ar}-\mathrm{H}), 8.03$ (d, $J=$ $7.8 \mathrm{~Hz}, 1 \mathrm{H}, \mathrm{Ar}-\mathrm{H}), 7.87$ (t, $J=7.2 \mathrm{~Hz}, 1 \mathrm{H}, \mathrm{Ar}-\mathrm{H}), 7.72$ (d, $J=7.8 \mathrm{~Hz}, 1 \mathrm{H}, \mathrm{Ar}-\mathrm{H}), 7.58(\mathrm{t}, J=7.2 \mathrm{~Hz}, 1 \mathrm{H}, \mathrm{Ar}-\mathrm{H}), 6.36$ (s, $1 \mathrm{H}, \mathrm{CH}), 4.88\left(\mathrm{t}, J=6.0 \mathrm{~Hz}, 2 \mathrm{H}, \mathrm{CH}_{2}\right), 3.77(\mathrm{t}, J=5.4$ $\mathrm{Hz}, 4 \mathrm{H}, \mathrm{CH}_{2}$ ), 3.01 (t, $\left.J=6.0 \mathrm{~Hz}, 2 \mathrm{H}, \mathrm{CH}_{2}\right), 2.69$ (s, 4H, $\left.\mathrm{CH}_{2}\right), 2.51\left(\mathrm{~s}, 3 \mathrm{H}, \mathrm{CH}_{3}\right) ;{ }^{13} \mathrm{C} \mathrm{NMR}\left(150 \mathrm{MHz}, \mathrm{CDCl}_{3}\right) \delta$ : $166.7,160.8,158.1,153.7,152.0,151.7,141.6,133.8$, $131.2,128.2,127.1,124.4,123.9,123.5,121.4,116.9$, 116.3, 115.7, 115.5, 67.0, 64.7, 57.2, 57.1, 54.1, 53.8, 18.6; MS (ESI) $m / z: 440.1\left([\mathrm{M}+\mathrm{Na}]^{+}\right)$. Anal. calcd for $\mathrm{C}_{24} \mathrm{H}_{23} \mathrm{~N}_{3} \mathrm{O}_{4}$ : C 69.05, H 5.55, N 10.07; found C 69.13, H 5.61, N 10.04 .

\subsection{6 抗肿瘤细胞毒性测试}

抗肿瘤细胞毒性采用改进的 Mosmann's MTT(噻唑 蓝)染色法测定 ${ }^{[23]}$. 具体操作如下: 于 96 孔板接种细胞 悬液, $90 \mu \mathrm{L}$ 孔, 细胞密度 $2 \times 10^{4}$ 个 $/ \mathrm{mL}, 37{ }^{\circ} \mathrm{C} ; 5 \% \mathrm{CO}_{2}$ 浓度的培养箱中过夜, 加 $10 \mu \mathrm{L} /$ 孔相应浓度药物 $(100 \mu \mathrm{mol} / \mathrm{L})$, 继续培 $44 \mathrm{~h}$, 加 MTT $10 \mu \mathrm{L} /$ 孔培养 $4 \mathrm{~h}$, 从培养箱中取出, 倒板, 加 DMSO $100 \mu \mathrm{L}$ 孔溶解 MTT, 测量 $570 \mathrm{~nm}$ 波长下肿瘤细胞 $\mathrm{OD}$ 值, 代入公式: $\left(\mathrm{OD}_{\text {control }}-\mathrm{OD}_{\text {treated }}\right) / \mathrm{OD}_{\text {control }} \times 100 \%$ 计算抑制率，顺铂 为阳性对照 ${ }^{[20]}$.

辅助材料(Supporting Information) 化合物 9 的核磁共 振氢谱和碳谱. 这些材料可以免费从本刊网站(http:// sioc-journal.cn/)上下载.

\section{References}

[1] Khan, I.; Zaib, S.; Batool, S.; Abbas, N.; Ashraf, Z.; Iqbal, J.; Saeed, A. Bioorg. Med. Chem. 2016, 24, 2361.

[2] Khan, I.; Ibrar, A.; Abbas, N.; Saeed, A. Eur. J. Med. Chem. 2014, 76, 193.

[3] Khan, I.; Ibrar, A.; Ahmed, W.; Saeed, A. Eur. J. Med. Chem. 2015, 90, 124.

[4] Ou, J. J.; Liu, K. C.; Wang, Y.; Zhang, H.; Liu, R. Q.; Li, Q. B.; Wang, Q. M.; Li, Y. Q.; Rui, C. H.; Liu S. Z. Chin. J. Org. Chem. 2014, 34, 526 (in Chinese).

(欧俊军, 刘克昌, 王毅, 张浩, 刘瑞全, 李奇博, 汪清民, 李永 强, 䒘昌辉, 刘尚钟, 有机化学, 2014, 34, 526.)

[5] Wang, Z.-Z.; Tang, Y. Tetrahedron 2016, 72, 1330.

[6] Kim, N. Y.; Cheon, C.-H. Tetrahedron Lett. 2014, 55, 2340.

[7] Yu, C. W.; Chang, P. T.; Hsin, L. W.; Chern, J. W. J. Med. Chem. 2013, 56, 6775. 
[8] Deepak, G.; Kumar, R.; Roy, R. K.; Sharma, A.; Ali, I. Med. Chem. Res. 2013, 22, 3282.

[9] Johannes, K.; Martens, J. Tetrahedron 2010, 66, 242.

[10] Unsworth, W. P.; Coulthard, G.; Kitsiou, C.; Taylor, R. J. K. J. Org. Chem., 2014, 79, 1368.

[11] Kametani, T.; Van LoC C.; Higa, T.; Koizumi, M.; Ihara, M.; Fukumoto, K. J. Am. Chem. Soc. 1977, 99, 2306.

[12] Xu, S.; Xu, S. P.; Li, L. M. Acta Pharm. Sin. 2002, 37, 113 (in Chinese).

(徐嵩, 徐世平, 李兰敏, 药学学报, 2002, 37, 113.)

[13] Hou, B. L.; Ai, Y.; Wang, C. L.; Zhang, N.; Yang, L.; Liu, Z. L.; Liu, J. L. Chin. J. Org. Chem. 2016, 36, 121 (in Chinese). (侯宝龙, 艾芸, 王翠玲, 张宁, 杨柳, 刘竹兰, 刘建利, 有机化 学, 2016, 36, 121.)

[14] Nacro, K.; Charles, Z.; Guzzo, P. R.; Herr, R. J.; Peace, D.; Frie- drich, T. D. Bioorg. Med. Chem. 2007, 15, 4237.

[15] Mayer, J. P.; Lewis, G. S.; Curtis, M. J.; Zhang, J. W. Tetrahedron Lett. 1997, 38, 8445.

[16] Chen, H.; Li, S.; Yao, Y. C.; Zhou, L. K.; Zhao, J. P.; Gu, Y. J.; Wang, K. R.; Li, X. L. Bioorg. Med. Chem. Lett. 2013, 23, 4785.

[17] Gunaratnam, M.; Swank, S.; Haider, S. M.; Galesa, K.; Reszka, A. P.; Beltran, M.; Cuenca, F.; Fletcher, J. A.; Neidle, S. J. Med. Chem. 2009, 52, 3774.

[18] Chen, G. S.; Kalchar, S.; Kuo, C.-W.; Chang, C.-S.; Usifoh, C. O.; Chern, J.-W. J. Org. Chem. 2003, 68, 2502.

[19] Venkata Sairam, K.; Gurupadayya, B. M.; Chandan, R. S.; Nagesha, D. K.; Vishwanathan, B. Curr. Drug Delivery 2016, 13, 186.

[20] Mosmann, T. J. Immunol. Methods 1983, 65, 55. 